\title{
Susceptibilidad y mecanismos de resistencia a insecticidas en Anopheles albimanus del sur de la Península de Yucatán, México
}

\author{
Felipe A. Dzul, Biól,(I) R. Patricia Penilla, PhD, (') Américo D. Rodríguez, PhD.(I)
}

\author{
Dzul FA, Penilla RP, Rodríguez AD. \\ Susceptibilidad y mecanismos de resistencia \\ a insecticidas en Anopheles albimanus del sur \\ de la Península de Yucatán, México. \\ Salud Publica Mex 2007;49:302-3II.
}

\section{Resumen}

Objetivos. Diagnosticar la resistencia a insecticidas y sus mecanismos en Anopheles albimanus del sur de la Península de Yucatán (PY), México. Material y métodos. La FI de An. albimanus colectados durante noviembre-diciembre de 2005 en seis localidades de los municipios Othón P.Blanco en Quintana Roo y Calakmul en Campeche, fue sometida a pruebas de susceptibilidad con deltametrina, DDT, pirimifos metil y bendiocarb, $y$ a pruebas bioquímicas para calcular los niveles de las enzimas involucradas en la resistencia. Resultados. An. albimanus fue resistente al DDT y a deltametrina en las seis localidades con niveles elevados de glutatión S-transferasas (GSTs), monooxigenasas y esterasas, y a pirimifos metil en La Unión con una alta frecuencia de la acetilcolinesterasa (AChE) alterada. Conclusión. Las poblaciones de An. albimanus colectadas al sur de la PY son resistentes al DDT y deltametrina, y en La Unión además al pirimifos metil, con mecanismos basados en la AChE alterada para el pirimifos metil, GST para DDT, y monooxigenasas y esterasas para piretroides. Los resultados del presente estudio tienen importantes consecuencias prácticas para el control químico de An. albimanus en el sur de la PY.

Palabras clave: resistencia a insecticidas; An. albimanus; Península de Yucatán; México
Dzul FA, Penilla RP, Rodríguez AD.

Susceptibility and insecticide resistance

mechanisms in Anopheles albimanus from the southern

Yucatan Peninsula, Mexico.

Salud Publica Mex 2007;49:302-3II.

\begin{abstract}
Objective. To diagnose susceptibility levels and insecticide resistance mechanisms in Anopheles albimanus from the southern Yucatan Peninsula (YP), Mexico. Material and Methods. FI generation of An. albimanus females, collected from November to December 2005 in six villages in the Othon P. Blanco municipality in Quintana Roo and the Calakmul municipality in Campeche, were exposed to deltamethrin, DDT, pirimiphos-methyl and bendiocarb in susceptibility tests, as well as to biochemical assays in order to calculate the enzyme levels related to insecticide resistance. Results. High levels of DDT and deltamethrin resistance were found in An. albimanus collected from the six villages, and a high resistance to pirimiphos-methyl was found in those from La Union, Quintana Roo. Biochemical assays showed high levels of glutathione S-transferase (GST), cytochrome P450 and esterases (with PNPA substrate) in all villages. The frequency of An. albimanus with altered acetylcholinesterase (AChE) was high in La Union (33\%). Conclusions. The An. albimanus populations collected in the south of the YP are resistant to DDT and deltamethrin, whereas resistance to pirimiphos-methyl was significant only in those collected from $\mathrm{La}$ Union. The mechanisms explaining this resistance are based on high concentrations of GST, cytochrome P450 and esterasas, the former being responsible for DDT metabolism and the others for pyrethroid metabolism. The altered AChE was the mechanism correlated to pirimiphos-methyl resistance in La Union. The results of the present study have important practical consequences for the chemical control of An. albimanus in the south of the YP.
\end{abstract}

Key words: insecticide resistance; An. albimanus; Yucatan Peninsula; Mexico

(I) Centro de Investigación de Paludismo, Instituto Nacional de Salud Pública, Tapachula, Chiapas, México.

Fecha de recibido: 28 de agosto de 2006 - Fecha de aprobado: 28 de mayo de 2007 Solicitud de sobretiros: PhD Rosa Patricia Penilla Navarro. Centro de Investigación de Paludismo, Instituto Nacional de Salud Pública, $4^{\mathrm{a}}$ Avenida Norte, esquina $19^{\mathrm{a}}$ Calle Poniente, Col. Centro, 30700 Tapachula, Chiapas, México. Correo electrónico: penilla@correo.insp.mx 
$\mathrm{E}$ paludismo es la enfermedad transmitida por vector más importante en el ámbito mundial debido a la mortalidad, morbilidad y carga de la enfermedad. Más de 100 países son afectados y la Organización Mundial de la Salud (OMS) estima que existen de 300 a 500 millones de personas infectadas anualmente y de éstas mueren entre 1 y 3 millones. ${ }^{1,2,3}$

En México, la Dirección General de Epidemiología reportó incidencias de paludismo de 2.77 a 7.27 casos por 100000 habitantes de 2000 a $2005^{4}$ y se estima que 30\% de la población se encuentra en riesgo. ${ }^{5}$ En la Península de Yucatán (PY) durante el periodo de 1990 a 2000 se reportaron en promedio más de 800 casos de paludismo. ${ }^{6}$ En la actualidad el sur de la PY es considerado de alto riesgo para la transmisión del agente causal de paludismo, ${ }^{7}$ debido a que comparte límites fronterizos con los países de Guatemala y Belice, lo cual aumenta el riesgo potencial de transmisión por el flujo incontrolado de las poblaciones humanas entre los países. ${ }^{8}$

En la PY se distribuyen tres especies de anofelinos; An. albimanus, An. pseudopunctipennis y An. vestitipennis, ${ }^{9-11}$ los cuales han sido implicadas en la transmisión del agente causal de paludismo en México. ${ }^{12-14}$ Aunque no se ha evaluado qué papel estén jugando en la transmisión de paludismo en esta zona, se sospecha que $A n$. albimanus es el principal vector. ${ }^{8}$ La medida de control de los anofelinos en México, se basó por más de medio siglo en la aplicación de DDT (1,1,1-trichloro-2,2-bis[ $p$ chlorophenyl]ethane), pero actualmente el programa de control de vectores de 2000-2006 contempla la aplicación de piretroides en los brotes y focos de transmisión. ${ }^{8}$

El uso intensivo y extensivo de insecticidas ha creado una presión de selección que ha favorecido el desarrollo y evolución de la resistencia en más de medio millar de artrópodos de importancia en salud pública, agrícola y veterinaria. ${ }^{15,16}$ En México se ha documentado la resistencia a insecticidas en los vectores de paludismo en la costa del Pacífico, ${ }^{17-22}$ sin embargo para la PY se desconocen los niveles de susceptibilidad a los insecticidas a pesar de ser recomendados por la OMS y la Norma Oficial Mexicana para la vigilancia epidemiológica, prevención y control de enfermedades transmitidas por vectores NOM-032-SSA-2002. ${ }^{23}$

El presente trabajo tuvo como objetivo generar información sobre los niveles de susceptibilidad de $A n$. albimanus a los cuatro grupos toxicológicos (organofosforados [OF], organoclorados [OC], carbamatos [CAR] y piretroides [PIR]) y determinar los mecanismos de resistencia de An. albimanus en el sur de la PY.

\section{Material y métodos}

Sitio de colecta. Se seleccionaron seis localidades del sur

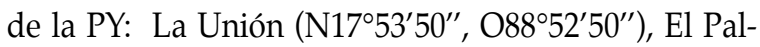

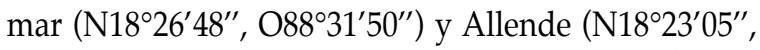
O88 $\left.33^{\prime} 15^{\prime \prime}\right)$, pertenecientes al municipio de Othón P. Blanco, Quintana Roo, y los ejidos Dos Laguna Norte (N18 $\left.53^{\prime} 34^{\prime \prime}, \mathrm{O}^{\circ} 9^{\circ} 21^{\prime} 03^{\prime \prime}\right)$, Nueva Vida (N18 $48^{\prime} 07^{\prime \prime}$,

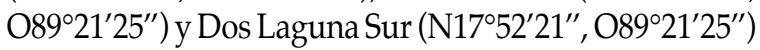
pertenecientes al municipio de Calakmul, Campeche. La selección de las localidades obedeció a que en los últimos años se han reportado casos de paludismo (comunicación personal del Departamento de Salud del municipio de Calakmul y Departamento de Vectores, J1-SSA Quintana Roo). Todas estas localidades son consideradas rurales y eminentemente agrícolas. ${ }^{24} \mathrm{El}$ clima de las localidades es cálido subhúmedo (Aw2), con dos periodos marcados, lluvias (mayo-octubre) y secas (noviembre-abril). ${ }^{25} \mathrm{La}$ temperatura media anual es de $24^{\circ} \mathrm{C}\left(22-26.6^{\circ} \mathrm{C}\right)$ y $25^{\circ} \mathrm{C}\left(24.4-27.8^{\circ} \mathrm{C}\right)$ en promedio, y la precipitación media anual es de $1052.8(552-1634) \mathrm{y}$ 1289.7 (793.5-2186.5) para las localidades de Campeche y Quintana Roo, respectivamente..$^{24} \mathrm{La}$ vegetación del sur de la PY es catalogada como selva mediana subperenifolia y selva alta perenifolia. ${ }^{26}$

Colecta de mosquitos. Los métodos usados en este trabajo fueron aprobados previamente por los comités de Ética, Bioseguridad e Investigación del Instituto Nacional de Salud Pública (INSP). Las colectas se realizaron a finales de noviembre y principios de diciembre de 2005. Las hembras de An. albimanus intentando picar fueron colectadas voluntariamente por los autores haciendo cebo humano $(\mathrm{CH})$ entre las 17:30 y 20:00 horas, así como aquellos mosquitos en reposo en corrales (CC) de Capra hircus, Bos taurus y Sus estrofa entre las 20:00 y 23:00 horas, con un esfuerzo de colecta de 0.55 y 0.75 horas / hombre, respectivamente. Los métodos utilizados de $\mathrm{CH}$ y CC aseguran capturar a mosquitos con diferentes hábitos alimenticios. Los mosquitos fueron colocados en recipientes de un galón de capacidad, cubiertos con malla mosquitera en la parte superior. El rango del horario de colecta incluye el pico de actividad de $A n$. albimanus ${ }^{27}$ y asegura que los mosquitos colectados reposando en corrales estén alimentados. Los mosquitos colectados en $\mathrm{CH}$ fueron alimentados con un animal del corral donde se efectuaron las CC colocando el recipiente con los mosquitos sobre el lomo o pierna del animal, previa autorización del propietario. La identificación de los mosquitos fue realizada in situ con la claves de Wilkerson y colaboradores, ${ }^{28}$ debido a que el material fue mantenido vivo y forzado a oviponer al tercer día 
para obtener la F1 y realizar pruebas de susceptibilidad, análisis bioquímicos y moleculares (datos no reportados en el presente trabajo). Una muestra del material $(n=10)$ incluyendo individuos de la F1 fue corroborado por especialistas en taxonomía de Anophelinae del Centro de Investigación de Paludismo (CIP) del INSP.

Obtención de la generación filial (F1) de An. albimanus. A los tres días de haberse colectado y alimentado, se realizó la oviposición forzada en 40 a 100\% de los mosquitos, y al resto se les proporcionó recipientes para que ovipositaran de manera natural. La técnica de oviposición forzada usada consiste en amputar manualmente un ala a las hembras previamente inmovilizadas a $-20{ }^{\circ} \mathrm{C}$ por aproximadamente 30 segundos, para posteriormente colocarlas individualmente en recipientes de plástico de $20 \mathrm{ml}$ de capacidad con papel filtro y agua para la oviposición. Esto permitió obtener la mayor cantidad de huevos y que la F1 fuera representativa de las localidades. Los huevos fueron filtrados con papel húmedo, y depositados en recipientes de $500 \mathrm{ml}$ con agua a $25^{\circ} \mathrm{C}$ para retrasar la eclosión de los huevos. El material biológico fue transportado al insectario del CIP y mantenido en condiciones de laboratorio $\left(26 \pm 2{ }^{\circ} \mathrm{C}, 70-80 \% \mathrm{HR}\right.$, agua destilada) para que los huevos eclosionaran y el desarrollo de las larvas, pupas y emergencia de adultos ocurriera de manera óptima.

Pruebas de susceptibilidad. Para cada localidad y tipo de colecta se realizaron pruebas de susceptibilidad con mosquitos de la generación F1 de un día de edad sin alimentar, siguiendo los procedimientos descritos por la OMS. ${ }^{29}$ Se introdujeron grupos de 25 mosquitos (hembras:machos, 1:1) en cada uno de los cuatro tubos de exposición recubiertos en su interior por papeletas (Whatman \# 1 de $12 \times 15$ ) impregnadas con la dosis diagnóstico (DD) de deltametrina $(0.025 \%)$, ${ }^{*} \mathrm{DDT}(4 \%)$, pirimifos metil $(0.9 \%$ ) y bendiocarb $(0.1 \%)$ por separado. Se utilizó el mismo número de mosquitos y tubos con papeletas sin insecticida para el control. Después de una hora de exposición, los mosquitos fueron transferidos a otros tubos con papeletas sin insecticidas; a las 24 horas se registró la mortalidad y se corrigió de acuerdo con la ecuación de Abbott. ${ }^{30}$

Ensayos Bioquímicos. Con el objeto de investigar los mecanismos de resistencia presentes en esta población, se utilizaron mosquitos individuales de la F1 de un día

\footnotetext{
* La dosis de 0.025 fue modificada recientemente a $0.05 \%$ por expertos de la OMS. En este estudio se decidió utilizar la primera DD con fines comparativos, debido a que todos los estudios de resistencia de los vectores de paludismo en México fueron realizados con la dosis antes de que fuera modificada.
}

de edad no alimentados (en grupos de 24 hembras y 23 machos, $n=47$ ) separados por localidad y tipo de colecta, a los cuales se les determinaron los niveles de glutatión S-transferasas (GSTs) en mmoles de clorodinitrobenceno conjugado $/ \mathrm{min} / \mathrm{mg}$ de proteína, esterasas con los substratos paranitrofenil acetato ( $\rho \mathrm{NPA}), \alpha-\mathrm{y} \beta$-Naftil acetato en $\mu$ moles del producto formado $/ \mathrm{min} / \mathrm{mg}$ proteína, acetilcolinesterasa alterada (AChE) en frecuencias del gen de resistencia y monooxigenasas (citocromos $\mathrm{P}^{450}$ ) en $\mu$ moles de citocromos $\mathrm{P}^{450} / \mathrm{mg}$ de proteína de acuerdo con la metodología descrita por Penilla y colaboradores. ${ }^{18}$ Se utilizó como referencia la cepa Panamá de $A n$. albimanus, la cual ha sido mantenida en laboratorio sin contacto de insecticidas por más de 30 años. Se consideraron individuos resistentes si sus valores estimados de las actividades de esterasas, monooxigenasas y GSTs fueron mayores a aquellos de la cepa Panamá. Para la AChE se consideró resistente cuando el porcentaje de inhibición de la AChE por el propoxur fue menor a $60 \%$, o a aquellos individuos que se salieron de la distribución normal de la población susceptible. Con estos datos se calcularon las frecuencias del gen de resistencia de la $\mathrm{AChE}$ asumiendo que las poblaciones se encontraban en equilibrio Hardy-Weinberg.

Análisis estadístico. Las gráficas se construyeron usando el software SPSS 14.0. Las diferencias entre los mosquitos colectados y los de la cepa Panamá se determinaron en análisis de varianza para las esterasas, GSTs y monooxigenasas y en pruebas de Kruskal-Wallis y de Student-Newmans-Keuls para la AChE. Se realizaron pruebas de $\mathrm{T}$ para determinar si existen diferencias en las actividades enzimáticas entre sexos y tipos de colecta (pruebas de U de Mann-Whitney para los datos de $\mathrm{AChE}$ ). Todas las pruebas se realizaron con un nivel de significancia de $95 \%$. Los análisis estadísticos fueron realizados con StatView ${ }^{\circledR 1992-1998 ~ S A S ~ I n s t i t u t e ~ I n c . ~}$

\section{Resultados}

Susceptibilidad a insecticidas en An. albimanus. Las pruebas de susceptibilidad mostraron niveles elevados de resistencia tanto al DDT como a la deltametrina en $A n$. albimanus colectados en las seis localidades del sur de la PY (cuadro I). En las localidades de Quintana Roo las poblaciones de An. albimanus presentaron importantes porcentajes de mortalidad que variaron entre 57 y $77 \%$ para la deltametrina, aunque para Allende fue sólo de $88 \%$, y entre 62 y $73 \%$ para el DDT, excepto por las colectas CC en El Palmar donde las mortalidades fueron sustancialmente altas (96\%) (cuadro I). Mientras que en Campeche se observaron porcentajes de mortalidades entre 77 y $82 \%$ con deltametrina y entre 53 y $60 \%$ con DDT. Con bendiocarb las mortalidades fueron de 
$100 \%$, excepto por mosquitos colectados con $\mathrm{CH}$ en $\mathrm{El}$ Palmar (99\%), mientras que para el pirimifos metil las mortalidades fueron mayores de $96 \%$, excepto para los mosquitos colectados en CC en La Unión (71\%) (cuadro I).

Análisis bioquímicos. Acetilcolinesterasa alterada. Los datos de actividad de la AChE de todas las localidades fueron significativamente diferentes entre sí y significativamente menores a los valores de la cepa Panamá
(Kruskal-Wallis; $\mathrm{H}=2$ 292.072; d.f=9; $p<0.0001$; StudentNewmans-Keuls, $p<0.05$ ) (cuadro II, figura 1). Las frecuencias del gen de resistencia de la AChE en todas las poblaciones de mosquitos colectadas oscilaron entre 3 y 33\% (cuadro II), siendo los mosquitos colectados en CC de La Unión los que presentaron las frecuencias más altas. Cuando se compararon los porcentajes de inhibición de la AChE por colecta dentro de cada localidad se encontraron diferencias significativas en Allende $(p=0.0063)$ y

Cuadro I

Resultados de LAS PRUebas de susceptibiLidad de LA OMS de AN. ALbiManus Colectados durante noviembre-diciembre de 2005 en Seis localidades del sur de la Península de Yucatán

\begin{tabular}{|c|c|c|c|c|c|c|}
\hline \multirow[t]{2}{*}{ Estado } & \multirow[t]{2}{*}{ Localidad } & Tipo de colecta & $D D T$ & Bendiocarb & Deltametrina & Pirimifos metil \\
\hline & & Cebo humano & $71.0 \pm 26.5$ & $100.0 \pm 0.0$ & $57.0 \pm 28$ & $98.9 \pm 2.1$ \\
\hline & \multicolumn{6}{|l|}{ La Unión } \\
\hline & & Corral & $67.0 \pm 31.8$ & $100.0 \pm 0.0$ & $57.8 \pm 12$ & $71.0 \pm 23.4$ \\
\hline \multirow[t]{5}{*}{ Quintana Roo } & & Cebo humano & $73.2 \pm 7.3$ & $99.0 \pm 2.0$ & $74.2 \pm 22.6$ & $96.0 \pm 0.0$ \\
\hline & \multicolumn{6}{|l|}{ El Palmar } \\
\hline & & Corral & $62.3 \pm 27.7$ & $100.0 \pm 0.0$ & $96.0 \pm 3.3$ & $100.0 \pm 0.0$ \\
\hline & Allende & Cebo humano & $88.0 \pm 9.8$ & $100.0 \pm 0.0$ & $76.8 \pm 24.5$ & $99.0 \pm 2.1$ \\
\hline & Dos Laguna Sur & Cebo humano & $59.7 \pm 28.3$ & $100.0 \pm 0.0$ & $81.8 \pm 9.7$ & $100.0 \pm 0.0$ \\
\hline \multirow[t]{2}{*}{ Campeche } & Dos Laguna Norte & Corral & $59.2 \pm 35.7$ & $100.0 \pm 0.0$ & $77.7 \pm 28.2$ & $96.9 \pm 6.25$ \\
\hline & Nueva vida & Cebo humano & $52.7 \pm 25.1$ & $100.0 \pm 0.0$ & $77.1 \pm 28.4$ & $100.0 \pm 0.0$ \\
\hline
\end{tabular}

Los valores son los porcentajes de mortalidad expresados como medias y sus desviaciones estándar

Cuadro II

Media \pm desviación estándar de las pruebas bioquímicas de An. albiManus Colectados durante NoViembre-diciembre de 2005 en SeIS localidades del sUR de la Península de YucatáN

\begin{tabular}{|c|c|c|c|c|c|c|c|c|}
\hline \multirow[t]{4}{*}{ Localidad } & Colecta & \multicolumn{2}{|l|}{ AChE } & \multicolumn{3}{|c|}{ Esterasas } & GSTs & Citocromos P450 \\
\hline & & \% de inhibición & fr & \multirow{2}{*}{\multicolumn{2}{|c|}{ Naftil acetato }} & & mmoles de CDNB & umoles de P450/ \\
\hline & & por el propoxur & gen & & & $\rho N P A$ & conjugado/min/ & $\mathrm{mg}$ de prot \\
\hline & & & & $\alpha$ & $\beta$ & & $m g$ de prot & \\
\hline \multicolumn{9}{|l|}{ Unión } \\
\hline & $\mathrm{CH}$ & $52.7 * \pm 21.4$ & 0.21 & $0.0003^{*} \pm 0.0001$ & $0.0003^{*} \pm 0.0001$ & $0.20 * \pm 0.16$ & $0.52 * \pm 0.33$ & $0.0008 * \pm 0.0006$ \\
\hline & $\mathrm{CC}$ & $49.4^{*} \pm 19.5$ & 0.33 & $0.0003^{*} \pm 0.0002$ & $0.0003^{*} \pm 0.0004$ & $0.35 * \pm 0.21$ & $0.46 * \pm 0.34$ & $0.0016 * \pm 0.0016$ \\
\hline \multirow[t]{2}{*}{ Palmar } & $\mathrm{CH}$ & $76.9 * \pm 08.9$ & 0.03 & $0.0005^{*} \pm 0.0003$ & $0.0004^{*} \pm 0.0003$ & ------------ & $0.50 * \pm 0.30$ & $0.0011 * \pm 0.0010$ \\
\hline & CC & $45.5^{*} \pm 19.6$ & 0.21 & $0.0004^{*} \pm 0.0005$ & $0.0006 \pm 0.0002$ & $0.31 * \pm 0.19$ & $0.69 * \pm 0.34$ & $0.0021 * \pm 0.0016$ \\
\hline \multirow[t]{2}{*}{ Allende } & $\mathrm{CH}$ & $51.7 * \pm 14.0$ & 0.09 & $0.0003^{*} \pm 0.0001$ & $0.0002 * \pm 0.0002$ & $0.24 * \pm 0.21$ & $0.45 * \pm 0.28$ & $0.0011 * \pm 0.0009$ \\
\hline & $\mathrm{CC}$ & $58.0 * \pm 11.3$ & 0.03 & $0.0004 * \pm 0.0004$ & $0.0004^{*} \pm 0.0002$ & $0.15 * \pm 0.08$ & $0.49 * \pm 0.24$ & $0.0012 * \pm 0.0007$ \\
\hline Dos Laguna Sur & $\mathrm{CH}$ & $58.2 * \pm \mid 4.7$ & 0.07 & $0.0002 * \pm 0.0002$ & $0.0003 * \pm 0.0002$ & $0.13 * \pm 0.14$ & $0.24 \pm 0.23$ & $0.0009 * \pm 0.0007$ \\
\hline Dos Laguna Norte & $\mathrm{CC}$ & $59.7^{*} \pm 08.5$ & 0.09 & $0.0004^{*} \pm 0.0002$ & $0.0002^{*} \pm 0.0002$ & $0.46 * \pm 0.29$ & $0.69 * \pm 0.25$ & $0.0011 * \pm 0.0007$ \\
\hline Nueva Vida & $\mathrm{CH}$ & $43.0 * \pm 09.5$ & 0.09 & $0.0005^{*} \pm 0.0002$ & $0.0004^{*} \pm 0.0002$ & ----------- & $0.62 * \pm 0.31$ & $0.0015 * \pm 0.0008$ \\
\hline Cepa Panamá & & $86.6 \pm 8.30$ & 0.00 & $0.0007 \pm 0.0002$ & $0.0006 \pm 0.0003$ & $0.04 \pm 0.02$ & $0.14 \pm 0.06$ & $0.0002 \pm 0.000 \mathrm{I}$ \\
\hline
\end{tabular}

* Indica que la media difiere del valor de la cepa Panamá con un nivel de significancia de 5\%. Las letras indican que existe diferencia entre colectas 

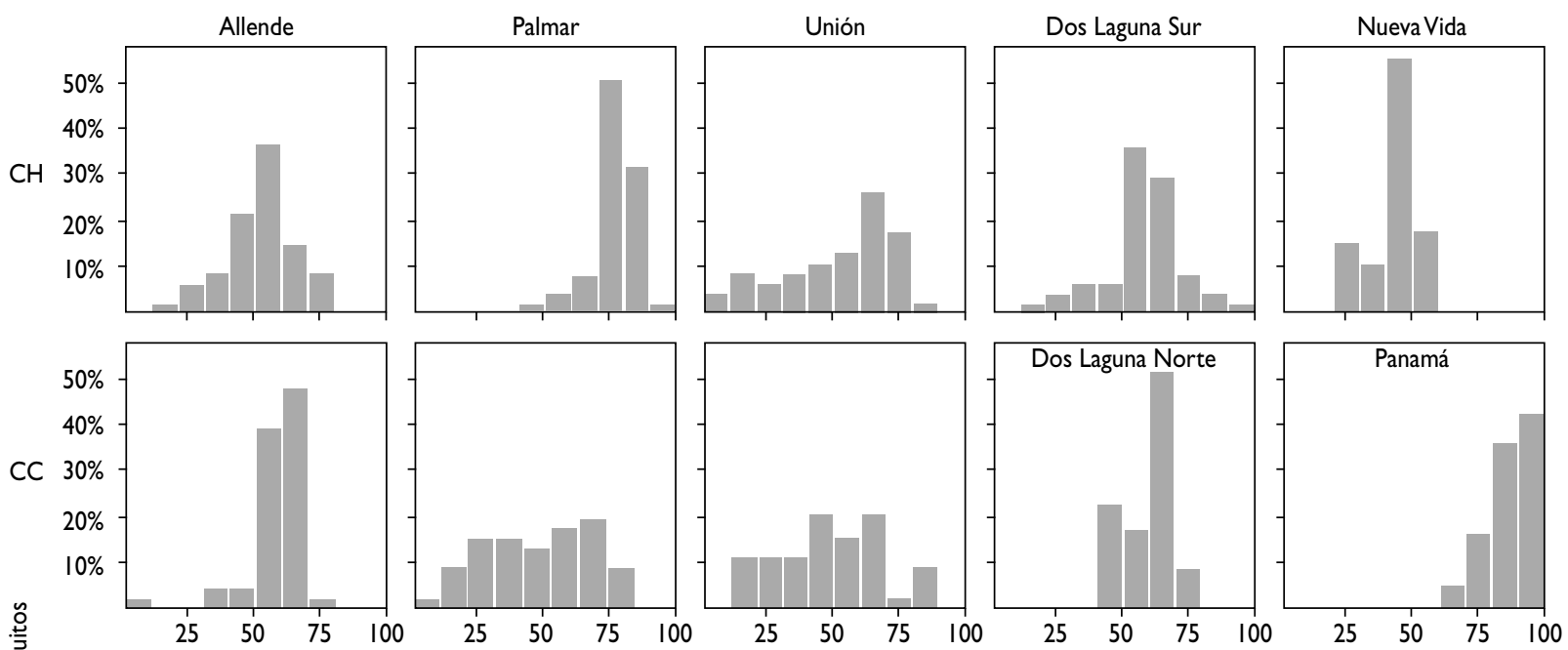

\% de inhibición de AChE por propoxur
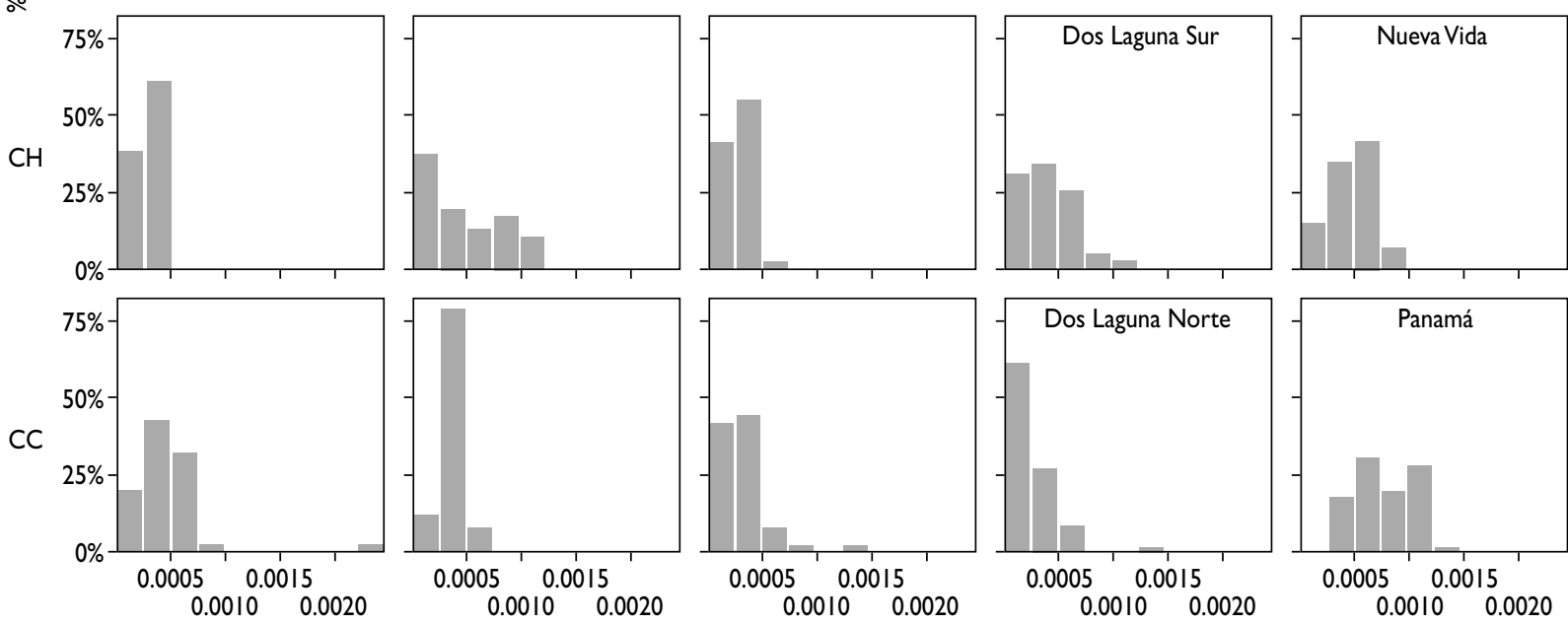

$\mu$ moles $\alpha$-Naftil $/ \mathrm{min} / \mathrm{mg}$ de proteína

Figura I. Porcentajes de inhibición de la AChE por el propoxur y actividad de esterasas Que utilizan $\alpha$-Naftil en an. albimanus colectados con cebo humano (CH) y en corrales (CC) en seis localidades del sur de la Península de Yucatán comparadas con la cepa Panamá

El Palmar $(p<0.001)$, siendo los mosquitos colectado en $\mathrm{CHy} \mathrm{CC}$, respectivamente, los que presentaron menores porcentajes de inhibición (cuadro II). Aunque las hembras presentaron valores de inhibición menores que las machos, estas diferencias fueron significativas sólo para CCen Dos Laguna Norte $(p=0.0293)$ y Palmar $(p=0.0045)$, y para $\mathrm{CH}$ en Allende $(p=0.0236)$.

Esterasas. En todas las localidades, $100 \%$ de individuos presentaron actividades de esterasas con los substratos alfa y beta-naftil acetato significativamente menores que la cepa Panamá (cuadro II, figuras 1 y 2) (ANDEVA, alfaF $=30.145 ;$ d.f $=425,9 ; p<0.0001$ : betaF=13.81; d.f=400, 9; $p<0.001$; Student-Newmans-Keuls, $p<0.05)$. Sin embargo, se observaron actividades significativamente elevadas de las esterasas con el sustrato $\rho N P A$ en todas las localidades comparadas con la cepa Panamá (ANDEVA; F=30.415; d.f=294, 9; $p<0.001$ ) (cuadro II, figura 2), siendo los mosquitos colectados en Dos 

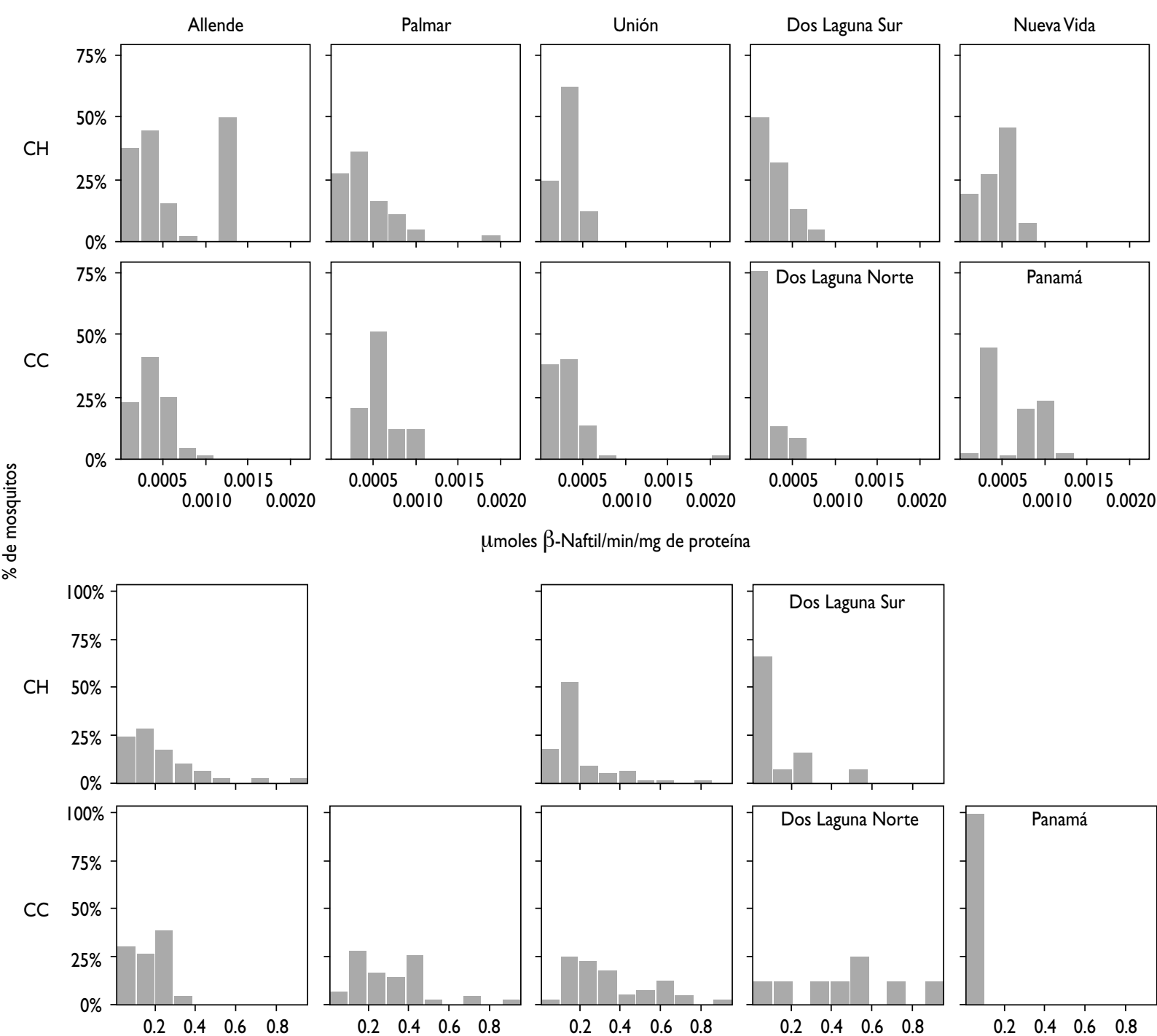

Actividad de $\rho N P A$

Figura 2.Actividad de esterasas Que Utilizan $\beta$-Naftil y $\rho$-NItrofenil acetato en An. albimanus colectados con cebo humano $(\mathrm{CH})$ y en corrales (CC) en seis localidades del sur de la Península de Yucatán comparadas con la cepa Panamá

Laguna Norte los que presentaron la mayor actividad. Las actividades enzimáticas de las esterasas ( $\rho$ NPA, $\alpha$ y $\beta$-Naftil acetato) fueron similares en ambos sexos y entre métodos de colecta.

GST y citocromos $P^{450}$. Los niveles de GSTs y citocromos $\mathrm{P}^{450}$ en los mosquitos analizados fueron significativamente diferentes entre las localidades, y mayores a los valores de la cepa Panamá (ANDEVA; GSTF=27.241; d.f=265, 8; $p<0.0001 ; \mathrm{P}^{450} \mathrm{~F}=23.933 ;$ d.f $=463,8 ; p<0.0001$;
Student-Newmans-Keuls, $p<0.05)$ (cuadro II, figura 3), excepto por lo valores de GST de Dos Laguna Sur. Las actividades de GSTs y el contenido de $\mathrm{P}^{450}$ en mosquitos de las localidades fueron entre 1.7 a 5 y 4.5 a 10 veces más altas que aquellos de la cepa Panamá, respectivamente. Los niveles de GSTs fueron similares entre sexos y métodos de colecta. Los niveles de citocromos $\mathrm{P}^{450}$ fueron mayores en los mosquitos adultos en CC que en $\mathrm{CH}$ en El Palmar y La Unión $(p<0.0001)$ (cuadro II). 

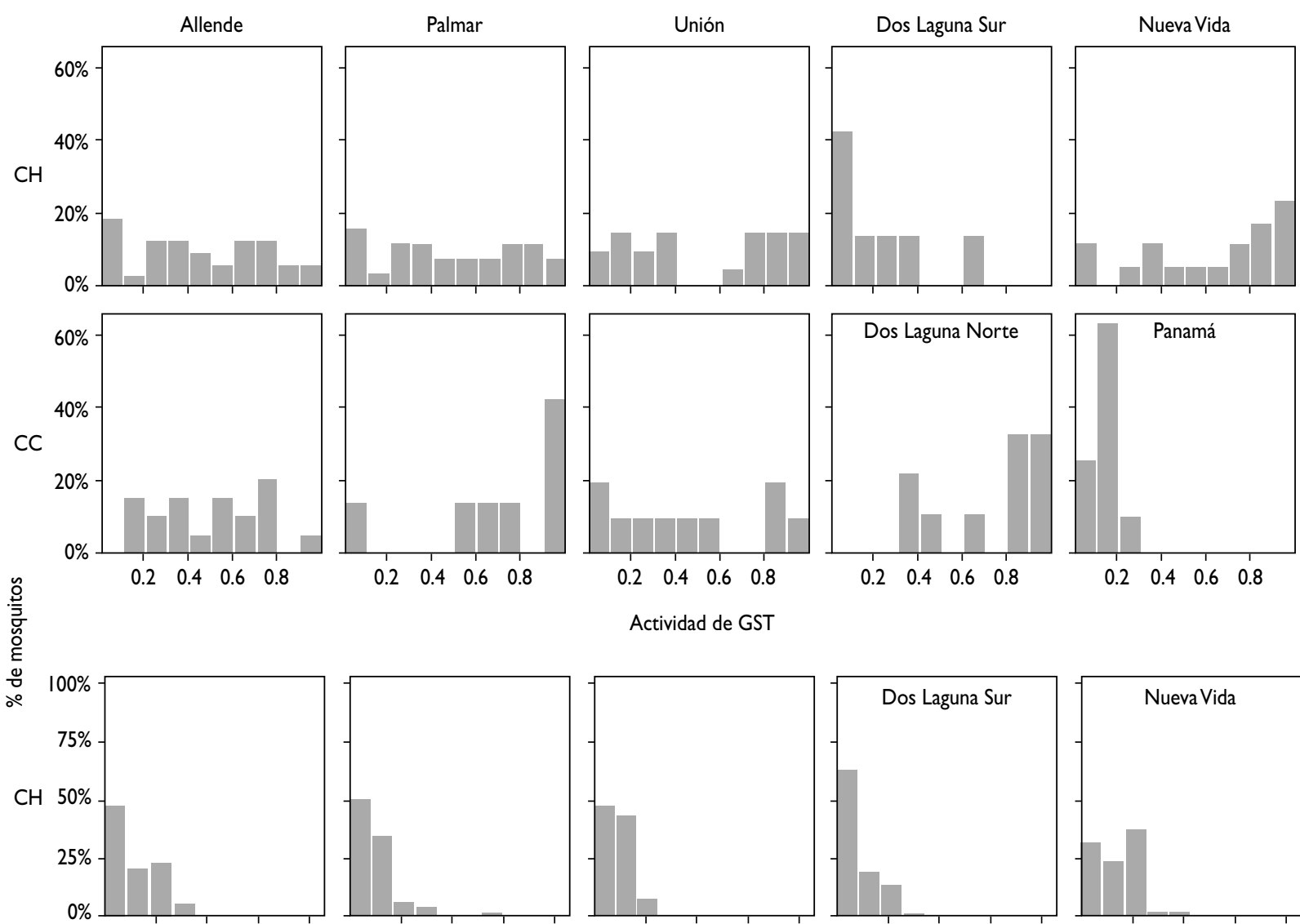

Actividad de GST
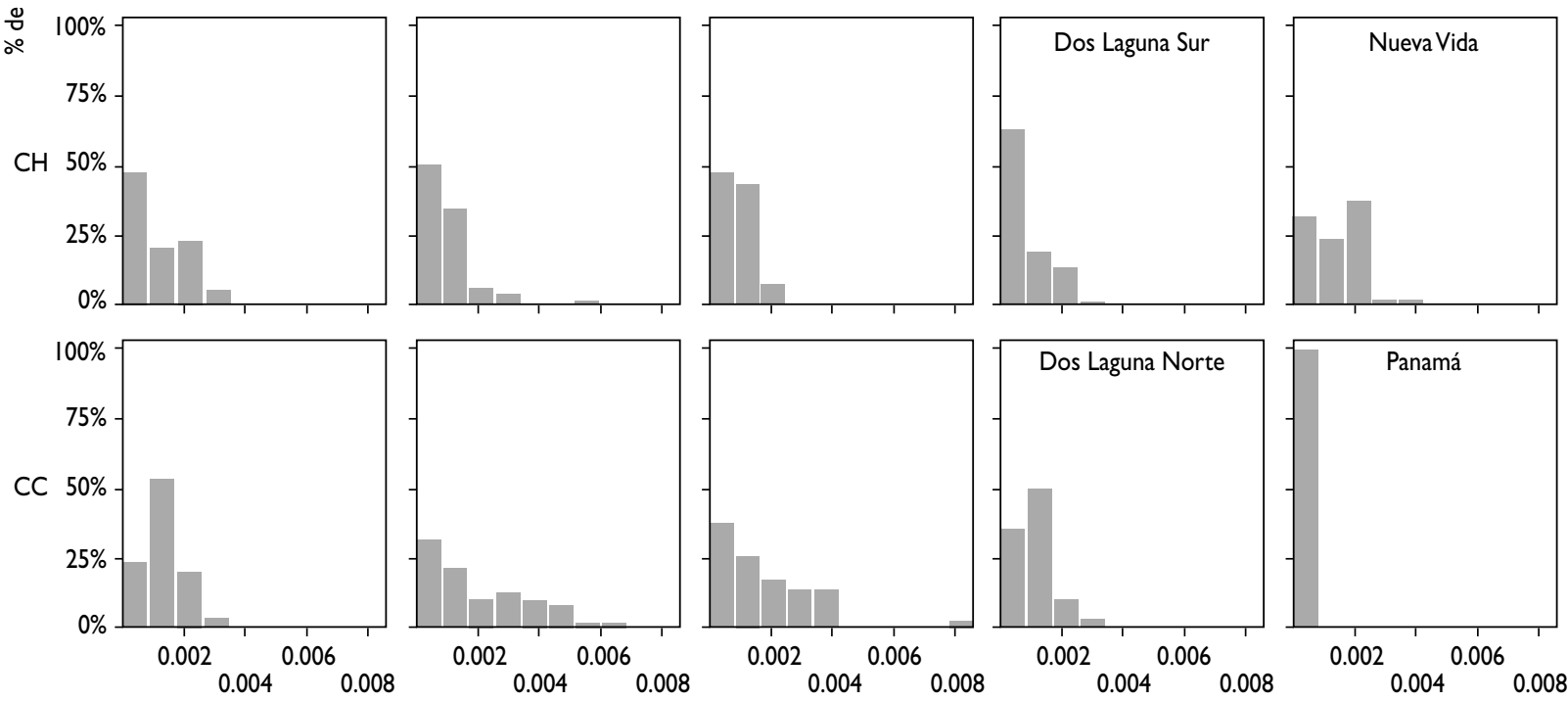

Umoles de Citromo P450/mg de proteína

Figura 3. Actividad de GSTs y contenido de Citoromos $P^{450}$ en An. albimanus colectados con cebo humano (CH) y en corrales (CC) en seis localidades del sur de la Península de Yucatán comparadas con la cepa Panamá

Las hembras de todas las localidades mostraron mayor contenido de $\mathrm{P}^{450}$ que los machos $(\mathrm{p}<0.0001)$.

\section{Conclusión}

En el presente estudio se determinó la susceptibilidad y mecanismos de resistencia a insecticidas en la generación $\mathrm{F} 1$ de An. albimanus colectados en el sur de la PY. Los resultados de las pruebas de susceptibilidad de la OMS indicaron resistencia a DDT y deltametrina en las seis localidades colectadas. La resistencia a estos insecticidas en An. albimanus también ha sido documentada en Tabasco ${ }^{19,22}$ y en los actuales focos residuales de transmisión de paludismo en el Estado de México, Sinaloa, Nayarit, Michoacán, Oaxaca y Chiapas. ${ }^{17,22}$ Los resultados de las pruebas bioquímicas confirmaron la resistencia a DDT y deltametrina, debido a que se observó que los niveles de las enzimas metabólicas de 
estos insecticidas, GSTs y citocromos $\mathrm{P}^{450}$, fueron significativamente superiores en los mosquitos colectados que en aquellos de la cepa susceptible Panamá. Los mismos mecanismos de resistencia al DDT y piretroides han sido descritos en An. albimanus en el sur de México ${ }^{18}$ y Guatemala. ${ }^{31}$ La detoxificación de piretroides por citocromos $\mathrm{P}^{450}$ también ha sido documentada en otras especies de mosquitos. ${ }^{32-34}$ Sin embargo, los elevados niveles de esterasas también podrían estar confiriendo resistencia a la deltametrina en menor grado como se ha observado que ocurre en An. albimanus, An. gambie, An. stephensi y Ae. aegypti. ${ }^{32,34}$ Estudios de metabolismo futuros confirmarían el papel que juegan estos dos mecanismos en la resistencia a piretroides.

Los resultados de las pruebas de susceptibilidad mostraron que An. albimanus es susceptible al bendiocarb y pirimifos metil en las seis localidades del sur de la PY, excepto la resistencia a este último insecticida observada en los mosquitos en la CC en La Unión (71\% de mortalidad). En general se ha observado que las poblaciones de An. albimanus colectados en las costas del Pacífico mexicano ${ }^{17,21}$ y en Tabasco ${ }^{22}$ son susceptibles a estos dos insecticidas, excepto por la resistencia a pirimifos metil en An. albimanus documentada en Chiapas. ${ }^{19,21}$ Las frecuencias de 3 a $33 \%$ de la AChE alterada indican que el gen de resistencia está presente en las seis localidades del sur de la PY, debido posiblemente a la presión ejercida por los OFs y CARs utilizados en control de plagas agrícolas. En México, el uso porcentual de estos dos grupos de insecticidas significa $45 \%$ de todos los plaguicidas usados para el control de plagas agrícolas, ${ }^{35}$ siendo el paratión metílico (37\%), el metamidofos $(10.6 \%)$ y el clorpirifos $(6 \%)$ los ingredientes activos más usados. ${ }^{36} \mathrm{El}$ desarrollo de resistencia a insecticidas en mosquitos por el uso intensivo y extensivo de insecticidas para el control de plagas agrícolas es un fenómeno ampliamente aceptado y documentado. ${ }^{37-39}$

Las diferencias en los niveles de susceptibilidad y actividad enzimática por método de colecta podría significar que hay diferente presión de insecticidas, es decir que el hecho que los mosquitos colectados con $\mathrm{CH}$ muestren más resistencia es debido probablemente a que se utiliza más insecticida para el control intra o peridomiciliar. Estas diferencias han sido detectadas en la costa de Chiapas, sin embargo, no son consistentes entre zonas estudiadas como ha sido observado en el presente estudio con AChE, debido a que en algunas localidades sí hay diferencias mientras que en otras no. ${ }^{18}$

En el presente estudio se observó mayor contenido de citocromos $\mathrm{P}^{450}$ en los mosquitos hembra cuando se comparó con los machos, lo cual ha sido reportado previamente en la costa de Chiapas. ${ }^{18,40}$ Los citocromos $\mathrm{P}^{450}$ son un sistema metabólico extremadamente importante en el crecimiento, desarrollo y reproducción de los insectos debido a que su inducción produce cambios hormonales a través de la síntesis y degradación de ecdiesteroides y hormonas juveniles, ${ }^{41,42}$ por lo tanto, es normal que las hembras presenten mayores niveles de citocromos $\mathrm{P}^{450}$ que los machos, debido a que la vitelogenesis y oogenesis son funciones exclusivas de las hembras.

Implicaciones en prácticas en el control de An. albimanus. Aunque el DDT ya no se esté aplicando para el control de vectores desde 2000 y su uso agrícola fue prohibido desde los años setenta, aún es posible detectar niveles elevados de resistencia a dicho insecticida en el sur de la PY. Evidencia similar ha sido documentada para An. pseudopunctipennis, An. albimanus y An. vestitipennis ${ }^{17,22}$ en los actuales focos residuales de transmisión de paludismo en México. La actual y amplia distribución de la resistencia al DDT en México podría indicar que la persistencia del DDT en el ambiente ejerce presión en las poblaciones de anofelinos o que la resistencia al DDT no tiene un costo en la adecuación de estas especies. Más investigación es necesaria para esclarecer este fenómeno.

En México el uso del DDT fue sustituido por piretroides después de haberse usado por casi medio siglo. En la actualidad la Norma Oficial Mexicana para la Vigilancia Epidemiológica, Prevención y Control de Enfermedades Transmitidas por Vector NOM-032-SSA-2002 sugiere a la deltametrina como primera opción seguida de lamdacialotrina, bifentrina, ciflutrina, y por último bendiocarb. ${ }^{23}$ Los resultados del presente estudio, en el que se demostró la resistencia a deltametrina y su correlación con el alto contenido de citocromos $\mathrm{P}^{450}$ y elevados niveles de esterasas (con sustrato $\rho \mathrm{NPA}$ ), sugieren que la eficacia del control de vectores con los insecticidas recomendados por la NOM-032 en las poblaciones mosquitos podría verse reducida. Los estudios sugieren que una buena opción de cambio podrían ser los CAR o el OF pirimifos metil, aunque cabe señalar que la sustitución de un insecticida a otro requiere de los estudios de susceptibilidad, ensayos bioquímicos (y/o moleculares), aceptabilidad y posibles efectos adversos ${ }^{43} \mathrm{y}$ los datos de morbilidad de la enfermedad para tomar una decisión acertada. Asimismo los datos sugieren que una estrategia de manejo de insecticidas (mezclas, mosaicos, rotaciones) es necesaria para implementar y mantener a largo plazo la susceptibilidad a pirimifos metil y bendiocarb, tratando de revertir la resistencia a deltametrina. 


\section{Agradecimientos}

A Gabriel Fuentes, Alma D. López, Francisco Solís, Octavia Pérez y Olga Ruth Gálvez por su ayuda técnica. A Eduardo Rebollar-Téllez y Pablo Manrique-Saide por su revisión crítica del escrito. El presente trabajo fue financiado por el proyecto de investigación 38897-M de CONACyT. Felipe Dzul fue becado por dicha institución para realizar estudios de posgrado en la Escuela de Salud Pública de México/INSP.

\section{Referencias}

I. World Bank. Rolling back Malaria, global strategy and booster program. Washington, DC; World Bank, 2005.

2. Breman JG,Alilio MS, Mills A. Conquering the intolerable burden of malaria:Whats news, whats hended: a summary.Am J Trop Med Hyg 200I;74(I):I2-16.

3. Snow RW, Guerra CA, Noor AM, Myint HY, Hay SI.The global distribution of clinical episodes of Plasmodium falciparum malaria. Nature 2005; 434:214-2I7.

4. Dirección General de Epidemiología.Anuarios de mortalidad y boletín epidemiológico. http://www.dgepi.salud.gob.mx/infoepi/index.htm. 5. Rodríguez MH, Hernández-Ávila JE, Betanzos-Reyes AF, Danis-Lozano R, González-Cerón L, Durán-Arenas LG.An ecosystem approach study of malaria transmission and control interventions in southern Mexico. Global Forum for Health Research 2004. Forum 8, México. Disponible en http://www.globalforumhealth.org/ Forum8/Forum8-CDROM/Posters/ Rodriguez\%20MH\%20F8-18I.doc.

6. Rodríguez DJ. Enfermedades transmitidas por vector en México. Rev Fac Med UNAM 2002; 43(3): 126-341.

7. Center for desease control and prevention (CDC). [Consultado:Febrero de 2005]. Disponible en http://www.cdc.gov/malaria/controlprevention/ mexico.htm.

8. Secretaría de Salud de México (SSA). Programa de Acción:

Enfermedades transmitidas por Vector. México, DF, 200I.

9. Vargas L, Martínez-Palacios A.Anofelinos Mexicanos:Taxonomía y distribución. CNEP-SSA. México: DF, 1956.

10. Días-Nájera A,Vargas L. Mosquitos mexicanos. Distribución geográfica actualizada. Rev Inv Salud Pública (Mex), 1973; 33: I I - 25.

II. Báñez-Bernal S, Martínez-Campos C. Claves de identificación de larvas de mosquitos comunes en las áreas urbanas y suburbanas de la República Mexicana (Díptera:Culícidae). Folia Entomol Mex 1994; 92:43-73.

12. Hoffman CC. On Anopheles pseudopunctipennis its relation to malaria in México. South. Med. J. 1932; 25(5):523-529.

13. Martini E. Los mosquitos de México. Boletines Técnicos de

Entomología Médica y Parasitología. Departamento de Salubridad. México, 1935; I (A): I-65.

14. Loyola EG,Arredondo-Jiménez JI, Rodríguez MH, Bown DN,VacaMarín MA. Anopheles vestitipennis, the probable vector of Plasmodium vivax in the Lacandon forest of Chiapas, México.Trans R Soc Trop Med Hyg 1991; 85: I7I-174.

15. World Health Organization.Vector resistance to pesticidas. Tech Rep series. $818,1992$.

16. Denholm I, Devine GJ,Williamson MS. Insecticide resistance on the move. Science 2002; 297:2222-2223.
17. Hemingway J, Penilla RP, Rodríguez AD, James BM, Edge W, Rogers $\mathrm{H}$, Rodríguez $\mathrm{MH}$. Resistance management strategies in malaria vector mosquito control.A large-scale field trial in southern Mexico. Pestic Sci 1997; 51:375-382.

18. Penilla RP, Rodríguez AD, Hemingway J,Torres JL,Arredondo-Jiménez $\mathrm{Jl}$, Rodríguez MH. Resistance management strategies in malaria vector mosquito control. Baseline data for a large-scale field trial against Anopheles albimanus in Mexico. MedVet Entomol 1998; 12: 217-233.

19. Penilla RP, Rodríguez AD, López AD, Solis F, Domínguez IA, Hemingway J, Rodríguez MH. Insecticide resistance in the Mexican malaria vectors from the transmission foci. In EMBO Workshop on the "Molecular and Population Biology of Mosquitoes and Other Disease Vectors"; 2005 Agosto; Kolymbari Chania, Crete.

20. Penilla P, Rodríguez A, Ranson H, Hemingway J, Rodríguez M. Research on malaria vector insecticide resistance in México. 4th International Congress of Vector Ecology. Reno, Nevada, October 2-7, 2005.

21. Penilla-Navarro P, Domínguez-Constantino IA, López-Solís AD, Solis-Santoyo F, Rodríguez-Ramírez AD. Diagnóstico de los mecanismos de resistencia a insecticidas en los mosquitos vectores de paludismo en Chiapas, Oaxaca y Tabasco, México. Entomología Mexicana, 2005; 4:763-767. 22. López-Solís AD, Solis-Santoyo F, Penilla-Navarro R, Rodríguez-Ramírez A. Resistencia a insecticidas en los mosquitos vectores de paludismo en Chiapas, Oaxaca y Tabasco, México. Congreso de Entomología. Tapachula, México. Entomología Mexicana, 2005; 4:768-772.

23. Secretaria de Salud de México (SSA). Norma Oficial Mexicana NOM032-SSA-2002, para la vigilancia epidemiológica, prevención y control de enfermedades transmitidas por vector. Publicada en el Diario Oficial de la Federación. Julio de 2003.

24. INEGl. http://www.inegi.gob.mx/geo/default.asp?c=124\&e=04, http://www.inegi.gob.mx/geo/default.asp?t=\&e=23\&c=124.

25. García E. Modificaciones al Sistema de Clasificación Climática de Koppen.Apuntes de Climatología. México, DF:Talleres Larios, 1973. 26. Flores JS, Espejel-Carvajal I.Tipos de Vegetación de la Península de Yucatán. Etnoflora Yucatanense. Mérida, México. Universidad Autónoma de Yucatán. 1994.

27. Frederickson EC. Bionomics and control of Anopheles albimanus. PAHO 1993. Technical paper 34

28. Wilkerson RC, Strickman D. Illustrated key to the female anophelinae mosquitoes of Central America and Mexico. J Am Mosq Control Assoc 1990; 6(I):7-34.

29. World Health Organization (WHO). Instructions for determining the susceptibility or resistance of adult mosquitoes to organochlorine, organophosphate and carbamate insecticides - diagnostic tests. Geneva, WHO, 198I; (unpublished document WHO/NBC/8I.806).

30. AbbottWS.A method of computing the effectiveness of an insecticida.J. Econ Entomol 1925; 18:265-267.

31. Brogdon WG, McAllister JC, Corwin AM, Cordon-Rosales C. Independent selection of multiple mechanisms for pyrethroid resistance in Guatemalan Anopheles albimanus (Diptera: Culicidae).J Econ Entomol 1999; 92(2):298-302.

32. Brogdon WG, McAllister JC. Insecticide resistance and vector control. Emerg Infec Dis, 1998; 4(4): 605-613.

33. Hemingway J, Ranson $\mathrm{H}$. Insecticide resistance in insect vectors of human disease.Ann Rev Entomol 2000; 45: 371-391.

34. Lui N, Xu Q, Zhu F, Zhang L. Pyrethroid resistance in mosquitos. Insect Science 2006, 13:159-166.

35. Secretaría de Salud/Secretaría de Medio Ambiente, Recursos Naturales y Pesca/Instituto de Naciones Unidas para la Formación Profesional e Investigaciones. Perfil nacional: Uso y manejo de las sustancias químicas en México. México; SSA/Semarnat/UNITAR:1997.

36. Asociación Mexicana de la Industria de Plaguicidas y Fertilizantes,A.C. (AMIPFAC).Anuario estadístico de la industria química mexicana. México; ANIQ. 1995, 1996. 
37. Mouchet J.Agriculture and vector resistance. Insect Sci Applic 1988; 9(3):297-302.

38. Lines JD. Do agricultural insecticides select for insecticide resistance in mosquitoes? A look at the evidence. Parasitol Today 1988; 4(7):SI7-20. 39. Diabate A, Baldet T, Chandre F, Akoobeto M, Guiguemde TR, Darriet F, Brengues C, Guillet P, Hemingway J, Small GJ, Hougard JM. The role of agricultural use of insecticides in resistance to pyrethroids in Anopheles gambiae s.l. in Burkina Faso. Am J Trop Med Hyg 2002 67(6):617-22

40. Villarreal-Treviño C. Flujo de genes de la metapoblación de Anopheles albimanus (Diptera: Culicidae) a pequeña escala en el ámbito microgeográfico de Chiapas y a gran escala en el ámbito macrogeográfico de México. (Tesis de Doctorado en Ciencias Biológicas). Universidad Autónoma de Nuevo León. Facultad de Ciencias Biológicas. 2001.

4I. Feyereisen R. Insect P450 enzymes. Ann Rev Entomol 1999; 44:507-533. 42. Scott JG,Wen Z. Cytocromes P450 of insect: the tip of the iceberg. Pest Manag Sci 200I; 57(10):958-67.

43. Rodríguez AD, Penilla RP, Rodríguez MH, Hemingway J,Trejo $A$ and Hernández-Ávila J. E.Acceptability and perceived side effects of insecticide indoor residual spraying under different resistance management strategies. Salud Publica Mex 2006; 48:315-322. 